\title{
Efficiency of Analytical Methodologies in Uncertainty Analysis of Seismic Core Damage Frequency*
}

\author{
Kenji KAWAGUCHI**, Tomoaki UCHIYAMA** and Ken MURAMATSU*** \\ ${ }^{* *}$ Computer Simulation \& Analysis of Japan Co., Ltd., \\ 1-3-9 Shibadaimon, Minato-ku, Tokyo 105-0012, Japan \\ E-mail: kawaguchi, uchiyama@csaj.co.jp \\ ${ }^{* * *}$ Center for Computational Science and E-systems, Japan Atomic Energy Agency \\ 5-1-5 Kashiwanoha Kashiwa-shi Chiba 277-8587, Japan \\ E-mail: muramatsu.ken@jaea.go.jp
}

\begin{abstract}
Fault Tree and Event Tree analysis is almost exclusively relied upon in the assessments of seismic Core Damage Frequency (CDF). In this approach, Direct Quantification of Fault tree using Monte Carlo simulation (DQFM) method, or simply called Monte Carlo (MC) method, and Binary Decision Diagram (BDD) method were introduced as alternatives for a traditional approximation method, namely Minimal Cut Set (MCS) method. However, there is still no agreement as to which method should be used in a risk assessment of seismic CDF, especially for uncertainty analysis. The purpose of this study is to examine the efficiencies of the three methods in uncertainty analysis as well as in point estimation so that the decision of selecting a proper method can be made effectively. The results show that the most efficient method would be BDD method in terms of accuracy and computational time. However, it will be discussed that BDD method is not always applicable to PSA models while MC method is so in theory. In turn, MC method was confirmed to agree with the exact solution obtained by BDD method, but it took a large amount of time, in particular for uncertainty analysis. On the other hand, it was shown that the approximation error of MCS method may not be as bad in uncertainty analysis as it is in point estimation. Based on these results and previous works, this paper will propose a scheme to select an appropriate analytical method for a seismic PSA study. Throughout this study, SECOM2-DQFM code was expanded to be able to utilize BDD method and to conduct uncertainty analysis with both MC and BDD method.
\end{abstract}

Key words: Seismic PSA, Uncertainty Analysis, Minimal Cut Set, Monte Carlo Simulation, Binary Decision Diagram, Core Damage Frequency

\section{Introduction}

Core Damage Frequency (CDF) defines the potential occurrence frequency of core damage accidents at Nuclear Power Plants (NPPs). Evaluating CDF and using it to regulate NPPs' activities are important processes because core damage accidents can lead to a massive release of radioactive materials into the environment. In fact, by using CDF, the potential risks of many existing NPPs have been assessed worldwide, especially since WASH- $1400^{1}$ successfully quantified the risks of NPPs in a comprehensive manner with 
Throughout the history of the risk assessments, it has been concluded by several studies that external events such as earthquake, fire, or flooding can have significant impacts on $\mathrm{CDF}^{2-4}$. To respond to this realization, the United Sates Nuclear Regulatory Commission (USNRC) required NPP utilities to perform the Individual Plant Examination of External Events (IPEEE) ${ }^{5}$ and issued a guide to help in conducting it ${ }^{6}$. In particular, seismic events have gotten a large amount of attention in these studies. In fact, the requirements of a widely-adapted standard for the risk assessments of NPPs (ASME/ANS RA-Sa-2009) ${ }^{7}$ were modified to include seismic CDF assessments. Then in 2011, caused by an earthquake and seismically induced tsunami, the Fukushima Daiichi Nuclear accident occurred, demonstrating the importance of seismically induced events. Therefore, the interest and urgency to evaluate CDF for seismic events is now increasing.

However, one of the main problems in assessing seismic CDF is that the well-established method to compute internal event CDF, called Minimal Cut Set (MCS) method, may not be adequately accurate for seismic CDF. This is because (1) in MCS method only approximates the CDF, (2) the accuracy of the approximation gets worse as the probability of each term in the cutset in computation becomes higher, and (3) the probability of each term in the cutsets are actually high in seismic CDF calculations in general. This problem has been recognized in the literature and hence the effects of the approximation on the mean value of CDF have been examined ${ }^{8-11}$.

Consequently, two alternative methods that have potential to solve this problem have been raised: Direct Quantification of Fault tree using Monte Carlo simulation (DQFM) method $^{12}$, or simply called Monte Carlo (MC) method in this paper, and Binary Decision Diagram (BDD) method ${ }^{13}$. However, these methods are still not used in most seismic CDF assessments. This is partially because the potential efficiencies of the two methods have not yet been well examined in the calculation of seismic CDF. One of the chief reasons why the effectiveness has not been comprehensively investigated might be that MC and BDD methods have not been studied together for seismic CDF, and hence there was no way to compare one of the methods with another reasonable method.

The lack of comparison studies on the methods can be especially true in uncertainty analysis. The uncertainty analysis shows, unlike point estimation, not only the mean value of CDF, but also the uncertainty distribution of CDF. The importance of uncertainty analysis has been recognized from the beginning of the risk assessment on NPPs. That is, WAHS $-1400^{1}$ introduced uncertainty analysis partly because a single value of CDF was thought to be inadequate for decision-making processes regarding NPPs activities ${ }^{13}$. The display of uncertainty in seismic CDF is particularly important because of its relatively high uncertainty in the seismic hazards and equipment fragility, in comparison with the uncertainty in internal event CDF as stated in the first Kashiwazaki Symposium on Seismic Safety of Nuclear Installations ${ }^{14}$.

Summarizing the above, while there are two relatively new methods that can solve the computational problem of the traditional method, the efficiencies of all the three methods need to be studied comprehensively, in particular for uncertainty analysis, so that an appropriate method for a PSA study can be selected. Indeed, there is no theoretical or empirical support as to which method should be used. Accordingly, previous studies have utilized each method without explaining why they chose certain ones over others.

In this study, the efficiencies of the traditional (MCS method) and the untraditional methods (MC and BDD methods) are examined in uncertainty analysis as well as point estimation of the seismic CDF. Based on the examinations, this paper will discuses as to which method can be used in accordance with the situations. Throughout this study, SECOM2-DQFM ${ }^{12}$ code was expanded to be able to utilize BDD method and to conduct uncertainty analysis with both MC and BDD method. 


\section{Analytical Methods to Evaluate Seismic Core Damage Frequency}

\subsection{Generic Procedure for All Methods}

This section is devoted to introducing the basic procedure used to evaluate seismic CDF that can be applied no matter which method (i.e. MCS, MC, or BDD method) is utilized. The outline of the general procedure and the focus of this study are illustrated in figure 1 . As indicated by the red box in the figure, the main interest of this study is the computation of CDF itself, not the overall procedure of Seismic Probabilistic Safety Assessment (PSA) involving hazard analysis, response analysis, etc. The general procedure and theory of an entire Seismic PSA are explained in Kennedy's influential paper ${ }^{15}$, and different approaches of fragility analysis are discussed in Hwang's review paper ${ }^{16}$. In the following, the general procedure is explained briefly, focusing on the computational procedures.

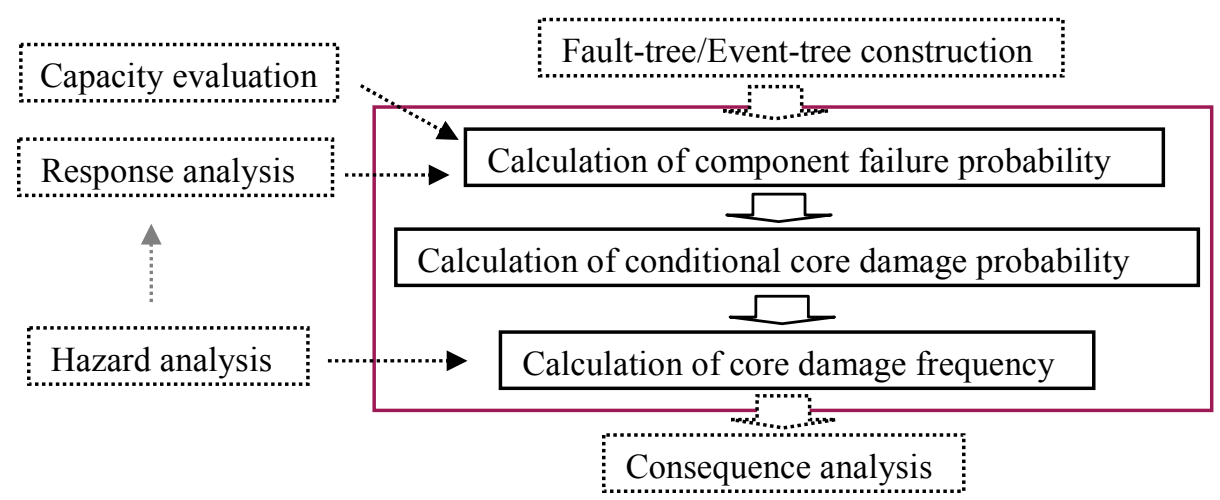

Fig.1 The outline of seismic PSA empathizing on the computational procedure

As in Fig. 1, the first step to assess seismic CDF is, in general, to construct Boolean functions for core damage events with Fault-tree and Event-tree. A Boolean Function is a function $f: x^{n}\{0,1\} \rightarrow\{0,1\}$, meaning that the combination of the states (0 or 1$)$ with regard to the $n$ number of components is transformed to a sate $(0$ or 1$)$. In the case of CDF calculation, $x$ represents a component of NPPs and $x_{i}=1$ means that the component $i$ is in the state of failure. Then, $f=1$ defines the situation where the core damage event occurs. Therefore, using the Boolean Function, potential combinations of component failures can be effectively transferred to the occurrence of core damage events. Boolean functions are usually constructed and represented by the Fault-tree and Event-tree in PSA. A comprehensive explanation of Boolean functions, Fault-tree and Event-tree can be found in Ref. (13).

After the Boolean functions are built, the probabilities of component failures are calculated. That is, the probability of $x_{i}=1$ is quantified for each component. This is usually represented by the probability that the response of a component exceeds the capacity of the component. Because the responses of a component differ in accordance to properties of seismic events, failure probabilities of a component are obtained for different seismic events. Usually, peak ground accelerations at bedrock are used to characterize seismic events, and hence failure probabilities of a component are computed for different peak ground accelerations at bedrock. As the gray line in Fig. 1 indicates, to obtain responses of components, response analysis can utilize the seismic events identified by hazard analysis as inputs ${ }^{17}$, but this is not always the case.

By using the Boolean functions for core damage events and component failure probabilities discussed above, the calculation of conditional core damage probability (CCDP) is conducted. CCDP is the total probability of core damage accidents occurring under the condition of certain peak ground accelerations at bedrock. The ways to quantify CCDP heavily depends on the method used; MCS, MC, or BDD method. Thus, the details of this process are discussed in the following sections explaining about each method. 
Finally, seismic CDF is computed by integrating CCDP with seismic hazard curves. Seismic CDF $F_{C D}$ is expressed by the following equation for all methods in this paper.

$$
F_{C D}=\int_{0}^{\infty} P_{C D}(\alpha)\left\{-\frac{d H(\alpha)}{d \alpha}\right\} d \alpha
$$

where $P_{C D}(\alpha)$ is CCDP at the given peak ground acceleration level $\alpha$, and $H(\alpha)$ is a seismic hazard curve which defines the frequencies of seismic events at the acceleration level $\alpha$.

\subsection{Minimal Cut Set Based method}

MCS method is the traditional and still most commonly used method to calculate CCDP in seismic PSA. In the PSA, MCS is a set of minimal combinations of component failures, which can cause the occurrence of an unwanted event, such as a core damage accident. Because of its nature, MCS itself is a very useful tool to review and document a PSA model. However, quantifying CCDP from MCS (i.e. MCS method) requires some form of approximation.

First, in order to obtain CCDP with MCS method, it is required to convert the Boolean functions into MCS. Then, the failure probability $P_{x}(\alpha)$ of each component $x$ in MCS is quantified with the assumption that the capacity and response of components are log-normally distributed as in Eq. (2) ${ }^{14,18,}$.

$$
P_{x}(\alpha)=\Phi\left[\frac{\ln (\bar{R}(\alpha))-\ln (\bar{S})}{\sqrt{\beta_{R}^{2}+\beta_{S}^{2}}}\right]
$$

where, $\Phi$ denotes cumulative distribution function for the standard normal distribution, $\bar{S}$ is the median value for the capacity of the component, and $\bar{R}(\alpha)$ indicates median value for the response of the component given ground acceleration $\alpha . \beta_{R}$ and $\beta_{S}$ express logarithmic standard deviations of $R$ and $S$ respectively. The right hand side of Eq. (2) is equivalent to the probability that $R$ exceeds $S$. By using component failure probabilities $P_{x}(\alpha)$, the probability of MCS $P_{M C S}(\alpha)$ is obtained simply by multiplying all $P_{x}(\alpha)$ in a minimal cut as in $P_{M C S}(\alpha)=\Pi\left[P_{x}(\alpha)\right]$.

After getting $P_{M C S}(\alpha)$, some approximations are required to compute CCDP. In seismic PSA, Upper Bound Approximation (UBA) method is generally applied because other approximation methods are less accurate or can exceed computational limits. Accordingly, this study utilizes UBA method. The UBA method can be expressed with the total number of MCS $k$ as the following.

$$
P_{T}(\alpha)=1-\prod_{i=1}^{k}\left[1-P_{M C S}(\alpha)\right]
$$

Eq. 3 can be directly utilized to obtain CCDP if the core damage event is expressed by only one Fault-tree. But normally, the core damage event is expressed with several accident sequences by using Event-trees. In that case, with the total number, L, of accident sequences resulting in core damage states, $\operatorname{CCDP} P_{C D}(\alpha)$ is expressed as in Eq. (3)'.

$$
P_{C D}(\alpha)=\sum_{j=1}^{L} P_{T_{j}}
$$

CCDP $P_{C D}(\alpha)$ obtained from Eqs. (3) and (3)' is accurate if and only if all $k$ MCS are independent from each other. However, this rarely happens in a practical PSA study and hence the effect of the approximation needs to be examined carefully. 
With the procedures discussed above and together with Eq. (1), the approximated mean value of CDF can be estimated by MCS method. However, to obtain uncertainty of CDF, it is necessarily to propagate uncertainty corresponding to capacity and response of components to CDF. To do so, the uncertainties are divided into two factors; uncertainty due to randomness and uncertainty derived from the lack of knowledge. Accordingly, logarithmic standard deviation of response and capacity are expressed as $\beta_{R}{ }^{2}=\beta_{R r}{ }^{2}+\beta_{R u}{ }^{2}$, $\beta_{S}{ }^{2}=\beta_{S r}{ }^{2}+\beta_{S u}{ }^{2}$, where $\beta_{R r}$ stems from randomness and $\beta_{R u}$ is from the lack of knowledge. By modifying equations appearing in Ref. (14) with those parameters and non-exceeding probability level $Z$, Eq. (2) can be expressed as the following for uncertainty analysis.

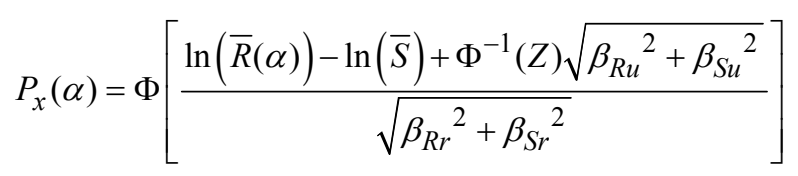

It is worth noticing that mean value of Eq. (4) is mathematically equivalent to the value of Eq. (3) ${ }^{18}$.

\subsection{Monte Carlo method}

In MC method, CCDP is assessed directly from the Boolean functions constructed with Fault-tree and Even-tree by means of Monte Carlo simulation. Unlike MCS method, this method does not need to use any approximation techniques, and hence this method holds certain accuracy in assessing CCDP or CDF, except for random number errors.

The method is conducted as the following. First, each component is determined to be 1 (failure) or 0 (intact) according to their failure probabilities such as in Eq. (2). Then, if core damage happens in the component configuration, it is counted and stored as the number of core damage events occurring. Finally, CCDP is calculated simply by dividing the counted total number of times that core damage occurs in the simulation by the total iteration number in the simulation.

As it could be realized from this procedure, MC method does not need to calculate the failure probabilities of components to quantify CCDP. Instead, all that is required is deciding whether the component is categorized as failure or intact during the trial of the simulation. Thus, instead of Eq. (2), the following equation can be utilized.

$$
\mathrm{Q}_{i}\left(\mathrm{R}_{i}, \mathrm{~S}_{i}\right)= \begin{cases}1 & \text { if } \mathrm{R}_{i}>\mathrm{S}_{i} \\ 0 & \text { otherwise }\end{cases}
$$

where, $\mathrm{Q}_{i}=1$ means that the component fails in the trial $i$, and $\mathrm{R}_{i}$ and $\mathrm{S}_{i}$ represent the response and capacity respectively in the trial. The values for the component response and capacity are in turn obtained with normally distributed random number $Y: \mathrm{R}_{i}=\bar{R} \exp \left(\beta_{R} \mathrm{Y}_{i}\right)$, $\mathrm{S}_{i}=\bar{S} \exp \left(\beta_{S} \mathrm{Y}_{i}\right)$. Note that with these equations, random numbers are generated separately for response and capacity, and hence correlations of response and capacity among components can be arbitrarily considered. Also, unlike other methods, the correlations can be considered in point estimation as well as uncertainty analysis by MC method. This particular approach for seismic CDF calculation was introduced as DQFM method ${ }^{12}$ and it is used in this paper. After calculating CCDP with these procedures, Eq. (1) is used to obtain CDF.

For uncertainty analysis, uncertainties of response and capacity are divided into uncertainties due to randomness and epistemic inadequacy in the same way as with MCS method. That is, $\mathrm{R}_{i}=\bar{R} \exp \left(\beta_{R r} \mathrm{Y}_{i}+\beta_{R u} \mathrm{Y}_{i}\right), \mathrm{S}_{i}=\bar{S} \exp \left(\beta_{S r} \mathrm{Y}_{i}+\beta_{S u} \mathrm{Y}_{i}\right)$, where each $\mathrm{Y}_{i}$ represents different random numbers and assumed to be independent from each other. Then, $M$ times simulation conducted by sampling $\mathrm{Y}_{i}$ for randomness $\left(\beta_{R r}\right.$ and $\left.\beta_{S r}\right)$ and by holding the same 
values for epistemic insufficiency $\left(\beta_{R u}\right.$ and $\left.\beta_{S u}\right)$ are performed $N$ times by changing the values of epistemic insufficiency.

Therefore, while other methods can conduct uncertainty analysis only by $N$ times computations $(\mathrm{O}(N))$, MC method needs to have $M$ by $N$ steps $(\mathrm{O}(M N))$. Another disadvantage of this method is that it contains random number errors, which become relatively high when very low probabilities contribute to the outcome of the computation. For this problem, dagger sampling was utilized ${ }^{19}$. However, for seismic CDF any special treatments for the problem would not be required because failure probabilities of components contributing to seismic CDF are likely to be high enough.

\subsection{Binary Decision Diagram method}

Binary Decision Diagram (BDD) is a well-known representation of Boolean functions, among others such as truth table, Karnaugh map, Ternary vector list, or Boolean Neural Networks. Compared to other representations, BDD representation has significant advantages in computing probability over Boolean functions. More concretely, exact CCDP and CDF can be obtained by BDD without any approximation (MCS method) and random number error (MC method) within a reasonable amount of time.

The first step of BDD method is to build BDD from a Boolean function by recursively applying Shannon decomposition to variables of the function. Let $F$ denote a Boolean function of $x$ among others, then

$$
F=x F[x \leftarrow 1]+x^{\prime} F[x \leftarrow 0]
$$

To illustrate how this step is conducted more concretely, let's assume that there are three variables, $x_{1}, x_{2}$, and $x_{3}$, all connected by the "OR" gate as shown in the far left of Fig. 2. If variable ordering is $x_{1}<x_{2}<x_{3}$, the first thing to do is to construct BDD with $x_{1}$ and $x_{2}$ (this means that the algorithm "Apply" proposed in Ref. (20) is utilized). Then, using Eq. (6) and some reduction rules, BDD for $x_{1}$ "OR" $x_{2}$ is built as in the middle of Fig. 2. Finally, the constructed BDD and $x_{3}$ are considered with connectivity "OR", and by applying Eq. (6), BDD which looks like the right side of Fig. 2 is generated.
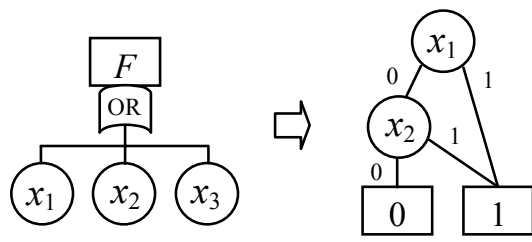

(OR)

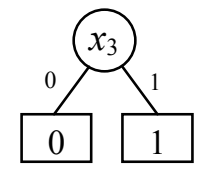

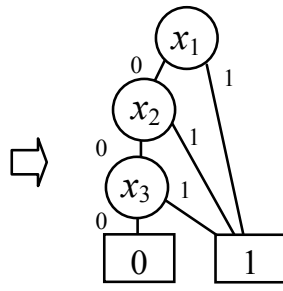

Fig. 2 An image of the BDD construction scheme

Calculating probability through BDD is relatively straightforward. Because BDD can be expressed by Eq. (6), the probability of $F$ is $P(F)=P(x) P(F[x \leftarrow 1])+P\left(x^{\prime}\right) P(F[x \leftarrow 0])$. For $P(x)$, Eq. (2) is applied in point estimation and $P\left(x^{\prime}\right)$ is simply $1-P(x)$. In uncertainty analysis, Eq. (4) is utilized as in MCS method. Like other methods, with CCDP obtained by using the above procedures, Eq. (1) is used to quantify CDF, but unlike other methods, CDF assessed by this method is an exact solution.

However, a major problem of BDD method is that it is not always possible to construct BDD from Boolean functions. This is because building BDD takes a lot of memory space, especially when Boolean functions, or a PSA model, are too big, such as the one from the nuclear industry as it is demonstrated in Table 7.4 of Ref. (13). Moreover, the size of BDD heavily depends on the processing order of Boolean functions' variables, and finding the best order is a NP-hard problem ${ }^{11}$. Therefore, a number of researchers have developed 
heuristics to find a reasonable order of variables. Though, it is not yet certain that BDD method can be used in all PSA models. Given this status of BDD method, several approximation methods were proposed to make sure that BDD could be made for all cases 21.

Summarizing discussions about different methods, while MCS method may not obtain accurate values of $\mathrm{CDF}, \mathrm{MC}$ and $\mathrm{BDD}$ methods are potentially able to do so. However, $\mathrm{CDF}$ quantified from $\mathrm{MC}$ method contains random number errors, and it would take a long time for uncertainty analysis. On the other hand, BDD method cannot always be utilized, depending on the structure of the PSA model, variable ordering, and selected heuristics.

\section{Calculations and Results}

\subsection{The Model}

The purpose of this study is to examine the efficiencies of analytical methods, instead of assessing the risk at a specific NPP. Given that objective, a hypothetical BWR plant PSA model $^{22}$ is utilized in this study because the model has been well examined and documented $^{8,9,12}$. This model consists of five initiating events and mitigation systems to ease accident progression induced by the initiating events.

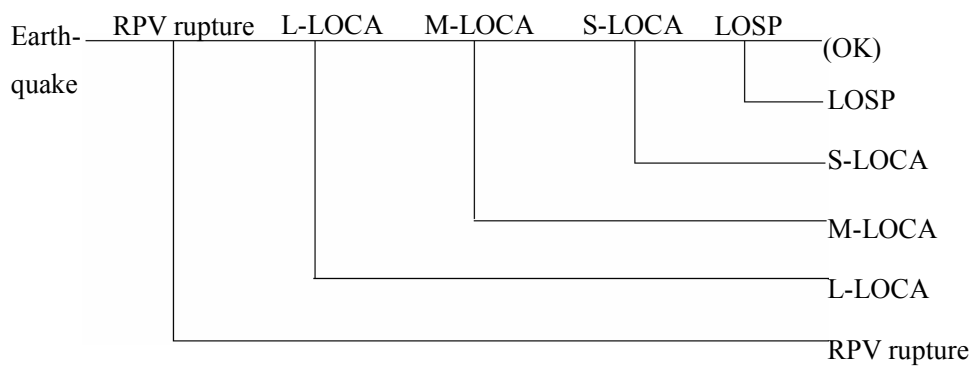

Fig. 3 The hierarchy event tree of the model

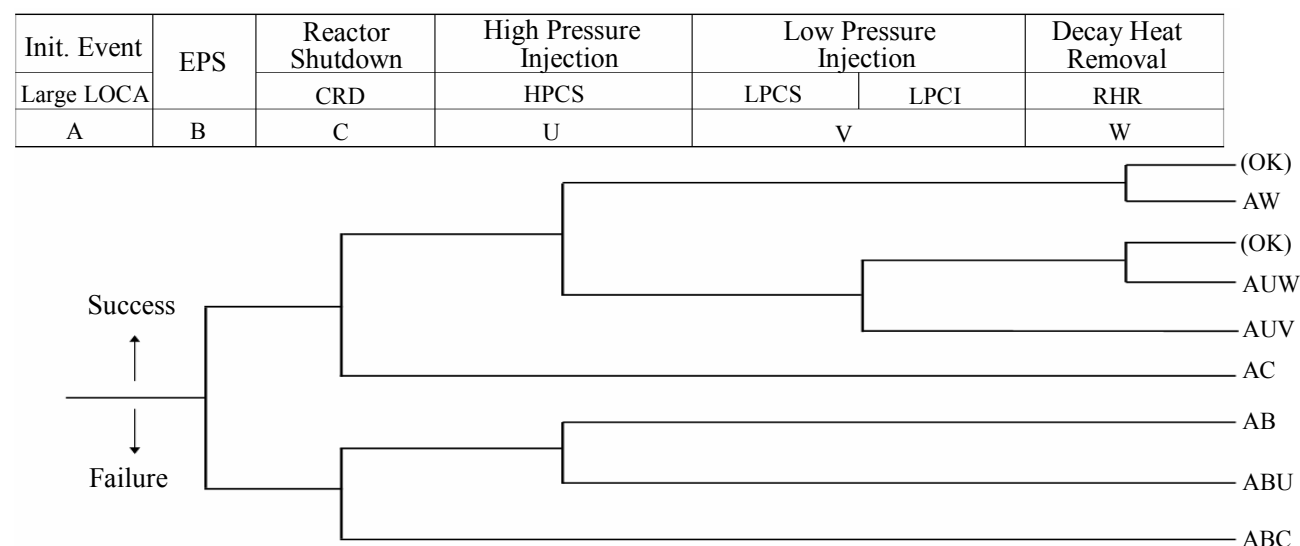

Fig. 4 The event tree for Large LOCA

The relationship of the five initiating events is considered by the hierarchy event tree as shown in Fig. 3. While the first initiating event in the figure, reactor pressure vessel (RPV) rupture, is assumed to immediately result in core damage, core damage due to the other four initiating events can be prevented by mitigation systems, such as emergency core cooling systems (ECCSs). For example, accident progressions occurring due to the Large Loss of Coolant Accident (LLOCA) are expressed by the event tree shown in Fig. 4. From the figure, it is known that if emergency power supply (EPS), reactor shutdown, and decay heat removal have succeeded, the accident paths lead to the end state "OK", meaning that core damage did not happen. Other end states, such as AW or AUW characterize each accident 
scenario that results in core damage. For instance, because "A" indicates LLOCA initiating event and " $\mathrm{W}$ " represents the failure of the residual heat removal (RHR) system, the accident sequence "AW" is an accident sequence where core damage happens due to the occurrence of LLOCA and the malfunction of RHR. The meanings of each character used in the end states or labels for all initiating events are shown in Table 1. Hereinafter, accident sequences will be indicated by the labels in accordance with Table 1 .

It was discussed above that if certain mitigation systems function properly, in some cases core damage can be avoided. In turn, whether or not mitigation systems can function to stop accident progressions depends on whether components consisting of mitigation systems can function or not. This dependency of mitigation systems on components is expressed by a Fault-tree. The Fault-tree is composed of 125 components, 66 'OR' gates, and 24 'AND' gates. The details of the Fault-tree and the parameters for component failure probabilities used in this study can be found in Ref. (22).

Table 1 The meaning of characters in accident sequence labels

\begin{tabular}{|cll|}
\hline Characters & \multicolumn{1}{c|}{ Abbrev. } & \multicolumn{1}{c|}{ Full Name } \\
\hline A & L-LOCA & Large Loss of Coolant Accident \\
\hline B & EPS & Emergency Power Supply \\
\hline \multirow{2}{*}{ C } & CRD & Control Rod Drive System \\
\cline { 2 - 3 } & SLCS & Standby Liquid Control Sysytem \\
\hline S1 & M-LOCA & Medim Loss of Coolant Accident \\
\hline S2 & S-LOCA & Small Loss of Coolant Accident \\
\hline T & LOSP & Loss of Offsite Power \\
\hline U 1 & RCIC & Reactor Core Isolation Cooling System \\
\hline U2 & HPCS & High Pressure Core Spray \\
\hline V & LPCI & Low Pressure Coolant Injection \\
\cline { 2 - 3 } & LPCS & Low Pressure Core Spray System \\
\hline W & RHR & Residual Heat Removal System \\
\hline X & DEP & Manual Depressurization \\
\cline { 2 - 3 } & ADS & Automatic Depressurization System \\
\hline
\end{tabular}

\subsection{Efficiency of Each Method in Point Estimation}

Before examining each method in uncertainty analysis, the efficiencies of the three methods in point estimation are briefly studied in order to illustrate the basic properties of the methods. The efficiency of the analytical method that this paper considers is two fold; accuracy and required computational time. This information is crucial in selecting one method over others. Therefore, to analyze the efficiency of each method, this paper compares and contrasts the accuracies of the results obtained and time used.

The CDFs quantified by each method are illustrated along with the iteration steps of MC method in Fig. 5 (a). The figure shows that the CDF calculated by MCS method did not agree with that by BDD method, which is the exact solution (around 2.22E-5). On the other hand, the $\mathrm{CDF}$ assessed by $\mathrm{MC}$ method converged to the exact solution around $10^{7}$ iterations. Though, Fig.5 (a) also illustrates that the CDF computed by MC method tends to be underestimated if the iteration number is not sufficiently large. To understand this property, let's assume that we have only one component that fails with the probability of 0.1. If Monte Carlo simulation is conducted only one time for this component, the estimated failure probability of the component is either 1.0 (overestimated) with a low probability $(0.1)$ or 0.0 (underestimated) with a high probability (0.9). This tendency remains until iteration is performed enough times. In this simple example, it is until we have 7 iterations that the probability of underestimating is $(1-0.1)^{7}<0.5$, and afterwards the result of MC method tends to be overestimated until 10 iterations, and from here there are chances to obtain true value, 0.1 . Note that this is only a tendency and the expected value of this component failure probability, for example, is always 0.1 regardless of the number of iterations. 
As another example, the occurrence frequency of sequence "S2UW" is shown in Fig. 5 (b). It is indicated in the figure that instead of overestimating, MCS method underestimated the occurrence frequency. Even in this case, MC method converged to the exact solution quantified by BDD method around $10^{7}$ iterations.

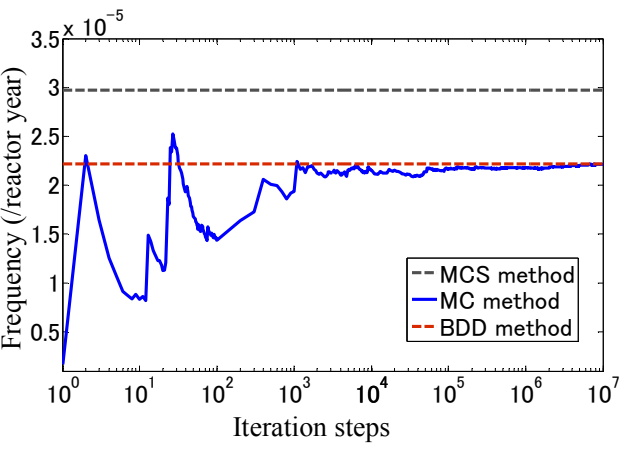

(a) CDF (the sum of all sequences)

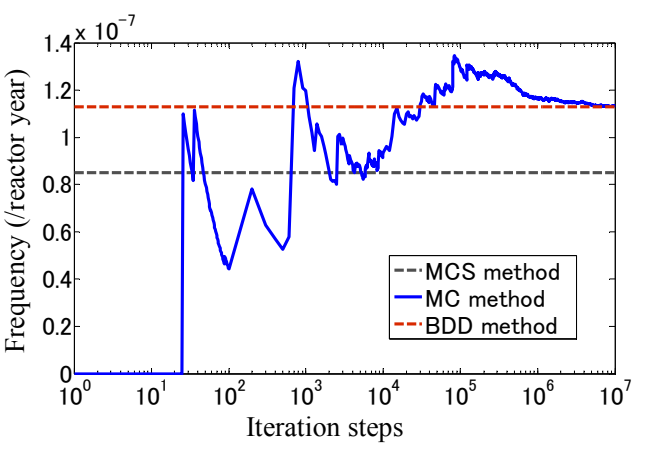

(b) Sequence "S2UW"

Fig. 5 Occurrence frequency obtained by each method along with sample sizes

The consistency of MC method with BDD method also held true in the accident sequence level as shown in Table 2. On the other hand, occurrence frequencies of some accident sequences obtained by MCS method were 2 times more or 0.9 times less than those assessed by BDD method. Therefore, by summarizing the above, it has been confirmed that MC method is almost as accurate as BDD method, but MCS method is not likely to be accurate enough in point estimation.

Table 2 The ratio of accident sequence occurrence frequencies to those by BDD method*

\begin{tabular}{|c|c|c|c|c|c|c|c|c|c|c|}
\hline \multicolumn{2}{|c|}{ Accident Sequence } & RPV & $\mathrm{AW}$ & AUW & AUV & $\mathrm{AC}$ & $\overline{\mathrm{AB}}$ & $\mathrm{ABU}$ & $\mathrm{ABC}$ & S1W \\
\hline \multirow{2}{*}{$\begin{array}{l}\text { The Ratio of } \\
\text { Frequency }\end{array}$} & MCS & 1.00 & 1.40 & 1.07 & 1.81 & 0.79 & 1.35 & 1.76 & 4.19 & 1.39 \\
\hline & $\mathrm{MC}$ & 0.97 & 1.01 & 1.00 & 1.01 & 1.01 & 1.00 & 1.00 & 1.00 & 1.01 \\
\hline \multicolumn{2}{|c|}{ Accident Sequence } & S1UW & S1UV & $1 \mathrm{UUX}$ & $\overline{\mathrm{S} 1 \mathrm{C}}$ & S1B & $1 \mathrm{BU}$ & S1BC & S2W & $\overline{2 \mathrm{U} 1 \mathrm{~W}}$ \\
\hline \multirow{2}{*}{$\begin{array}{l}\text { The Ratio of } \\
\text { Frequency }\end{array}$} & MCS & 1.09 & 1.73 & 0.92 & 0.82 & 1.33 & 1.78 & 3.89 & 1.28 & 1.43 \\
\hline & $\mathrm{MC}$ & 0.99 & 1.00 & 0.9 & 0.98 & 1.01 & 1.00 & 1.00 & 0.99 & 1.02 \\
\hline \multicolumn{2}{|c|}{ Accident Sequence } & $2 \overline{\mathrm{UW}}$ & 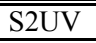 & $\overline{\text { S2UX }}$ & $\overline{\mathrm{S} 2 \mathrm{C}}$ & $\overline{\mathrm{S} 2 \mathrm{~B}}$ & 2BU1 & S2BU & $2 \mathrm{BC}$ & $\overline{\mathrm{TW}}$ \\
\hline \multirow{2}{*}{$\begin{array}{l}\text { The Ratio of } \\
\text { Frequency }\end{array}$} & MCS & 0.75 & 1.11 & 1.20 & 0.87 & 1.31 & 1.36 & 1.96 & 3.42 & 1.22 \\
\hline & $\mathrm{MC}$ & 1.00 & 0.99 & 1.04 & 0.99 & 1.00 & 1.00 & 1.00 & 1.00 & 1.01 \\
\hline \multicolumn{2}{|c|}{ Accident Sequence } & TU1W & TUW & TUV & TUX & TC & TB & TBU1 & TBU & TBC \\
\hline \multirow{2}{*}{$\begin{array}{l}\text { The Ratio of } \\
\text { Frequency }\end{array}$} & MCS & 1.30 & 0.92 & 1.20 & 1.16 & 1.25 & 1.22 & 1.23 & 1.75 & 2.62 \\
\hline & $\mathrm{MC}$ & 1.00 & 0.99 & 1.00 & 0.96 & 1.02 & 0.99 & 0.98 & 0.99 & 1.00 \\
\hline
\end{tabular}

* The ratios of cells colored by gray are less than $90 \%$, and the proportions of cells colored by orange are more than $200 \%$.

However, another important question is how long each method takes to obtain the results with this degree of accuracy. For the experiment above, MCS and BDD methods took 3.5 and 1.2 seconds respectively, while MC method spent 14,248 seconds. These times were observed using a personal computer running Windows 7 with a processor speed of 3.1 $\mathrm{GHz}$ and $4 \mathrm{~GB}$ of RAM. Therefore, as far as this PSA study is concerned, BDD method turned out to be the most efficient in point estimation in terms of accuracy and computational time. But, as discussed in section 2.4, the main concern of BDD method is not computational time, but time complexity and memory space, which varies from model to model and variable ordering. Because the time complexity and required memory space can exceed the computer's resources, BDD simply cannot be constructed in some cases while MC method is always applicable in theory. Therefore, the results above show that if BDD is not applicable to a PSA study, one should think about the accuracy-time tradeoff to select MC method or MCS method. 


\subsection{Efficiency of Each Method in Uncertainty Analysis}

In order to study the efficiencies of MCS, MC and BDD methods in uncertainty analysis, this section first compares CCDP curves, and then examines CDFs calculated with two different hazard curves. The Monte Carlo simulation was conducted $10^{5}$ times for MCS and BDD methods, and $10^{5}$ by $10^{4}$ times for MC method.

The comparison of CCDP curves computed by MCS, MC, and BDD methods is shown in Fig. 6. The figure shows that the CCDP curves of MC method and BDD method highly agreed with each other and that the curve of MCS deviated from others. More concretely, the CCDP curves corresponding to $95 \%, 50 \%, 5 \%$ and the mean values were overestimated by MCS method. In particular, the $95 \%, 50 \%$ and mean values of CCDP calculated by MCS method exceeded 1.0 which should not go beyond 1.0 in theory. This happened because each of the sequence occurrence probabilities (Eq. (3)), the sum of which is CCDP (Eq. (3)'), can incorrectly reach up to 1.0 due to the overestimation of UBA method and the imperfect handling of negation. Essentially, treating negation correctly can prevent this bizarre phenomenon because the correct treatment of negation makes sure that all sequences are mutually exclusive. But, of course, the treatment of negation is something that cannot be done perfectly in the MCS method ${ }^{10}$.

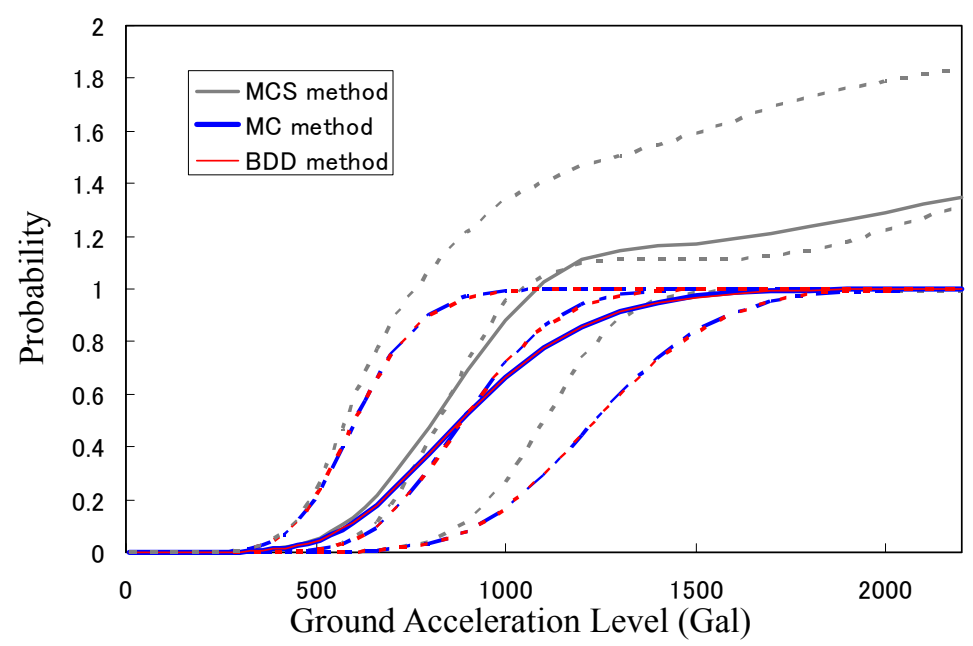

Fig. 6 CCDP curves: from the top to the bottom, $95 \%, 50 \%$, and $5 \%$ values are plotted with dotted lines and mean values are expressed with solid lines

In addition to the overall overestimation of the MCS method, the relationship of the mean curve and the median (50\%) curve obtained by MCS method turned out to be different from the relationships computed by other methods. For MC and BDD methods, the relationship changed only once; the mean curve is higher than the median curve until a certain point (around $900 \mathrm{Gal}$ ), and afterward the mean curve becomes lower. The cross point seems to be closely related to the point where the $95 \%$ curve reaches 1.0 . This is because the mean value can be higher than the median value when the right tail of the distribution of CCDP is longer than the left side of the distribution, and because when the $95 \%$ curve reaches 1.0 , the right tail of the distribution is more likely to be limited by the value 1.0. On the other hand, the relationship of the mean and median curves for MCS method changes twice instead of only once. The fist change occurs around $800 \mathrm{Gal}$ when the increment of $95 \%$ value starts decreasing and the second intersection appears when the increment of the $95 \%$ value starts increasing again. The change on the increment of $95 \%$ can happen twice because of the following reasons. As discussed above, the effect of the probabilistic limitation (1.0) can take place on all accident sequences in MCS method, 
instead of only on a total CCDP. Therefore, when the $95 \%$ values of some sequences reach 1.0 , the increment of $95 \%$ value decreases, but afterward the $95 \%$ values of other sequences can increase rapidly at some larger ground acceleration levels, which resulted in the two cross points of the mean and median curves.

For uncertainty analysis of CDFs, two calculation cases corresponding to different hazard curves were considered. One of the curves was utilized to examine the efficiencies of all three methods. Another curve was employed as a hazard curve containing realistic uncertainty information to compare the approximated results (by MCS method) and the exact solutions (by BDD methods).

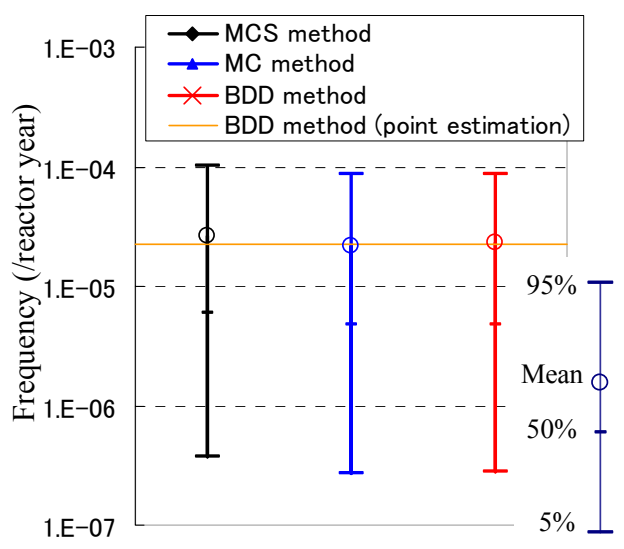

(a) CDF distributions

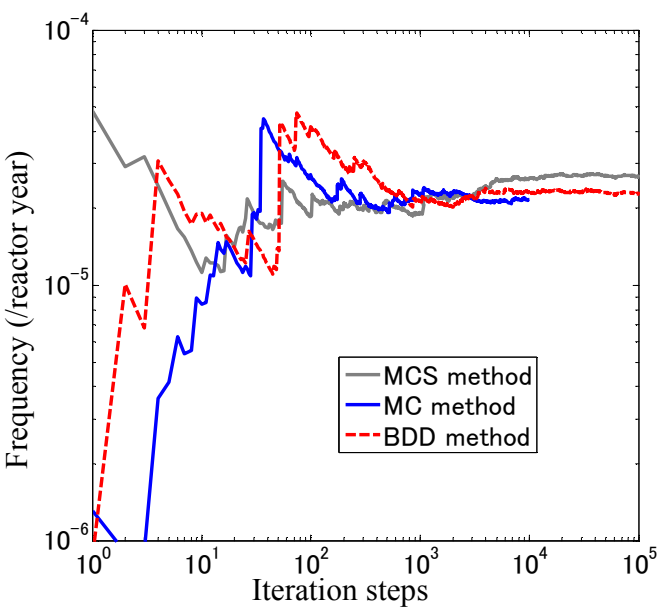

(b) CDF convergence trajectories

Fig. 7 The results of uncertainty analysis with the fist hazard curve

The first hazard curve utilized is the same one that was presented with the hypothetical BWR plant PSA model in Ref. (22) and used in the point estimate calculation of the previous section. The uncertainty of this hazard curve was represented by the logarithmic standard deviation $\beta=1.4$ for all ground acceleration levels ${ }^{22}$. The results of the uncertainty analysis by MCS, MC and BDD methods are shown in Fig. 7 (a). As indicated in the figure, the uncertainty ranges, or the $90 \%$ confidence intervals, took place within the order of $10^{-7}$ to around $10^{-4}$ for all methods. The comparison of results shows that the results of uncertainty analysis of MC and BDD methods are consistent with each other, while that of MCS again disagreed with the other two methods. However, the degree of deviation with regard to the mean value by MCS method from the other methods was less compared with that in the point estimate calculation; while the ratio of the mean value obtained by MCS method to that by BDD method was 1.34 in the point estimation, the ratio became 1.17 in the uncertainty analysis. This phenomenon can be explained by the fact that the probabilities for each accident sequence cannot exceed 1.0 and hence the right tail of the distribution for CDF cannot be overestimated as much as the value of point estimation. In fact, although the exact same random numbers were used for MCS and BDD methods, Fig. 7 (b) shows that while intense variations towards unreasonably high values were occurring in BDD method (around $10^{2}$ iterations), it was not happening in MCS method, which supports the argument above.

The mean values of CDFs reached the point of convergence around $10^{4}$ iterations for all MCS, MC, and BDD methods (Fig. 7 (b)). But, for MC method, in order to perform each of $10^{4}$ iterations presented in Fig. 7 (b), it was required to conduct $10^{5}$ iterations because MC method utilizes Monte Carlo simulation to compute each value of CDF as well as the distribution of CDF. Consequently, MC method took a relatively large amount of time to obtain the result presented above. That is, the amount of time spent by MC method was 1,354,669.3 seconds (around 376 hours) while those by MCS and BDD methods were 
32,864.8 seconds (around 9 hours) and 19,636.6 seconds (around 5 hours) respectively.

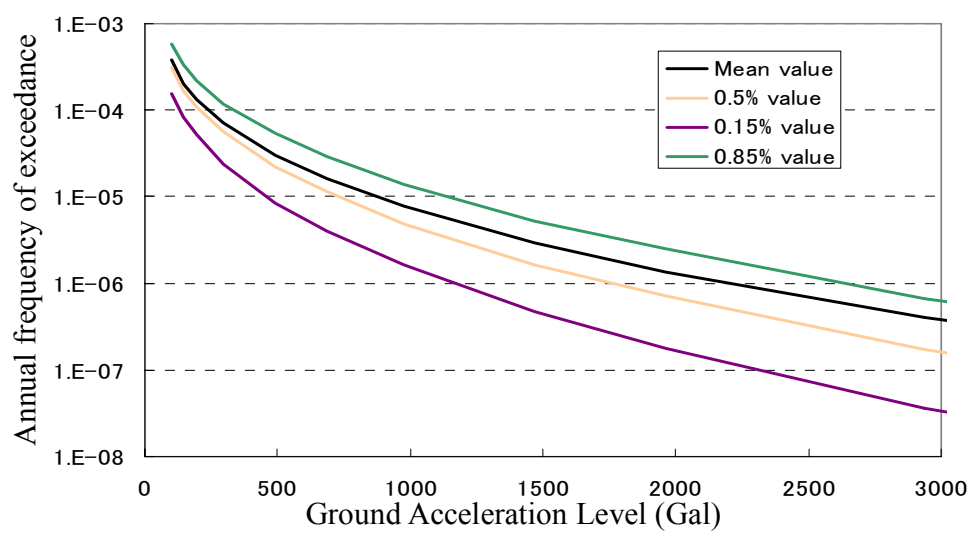

Fig. 8 The hazard curve for the second experiment

Another set of hazard curve (Fig. 8) used in this paper comes from the CEUS-SSC (Central and Eastern United States - Seismic Source Characterization) project ${ }^{23}$. In this project, a new seismic model was developed, and it will be used for seismic re-evaluations in response to Fukushima Daiichi nuclear accident as well as for licensing-purpose assessments. To demonstrate the calculation of the model, the Electric Power Research Institute (EPRI), the U.S. Department of Energy (DOE), and USNRC published a set of hazard curves at the Manchester site, which is utilized in this paper as a recent and rigorously calculated hazard curves with uncertainty information.

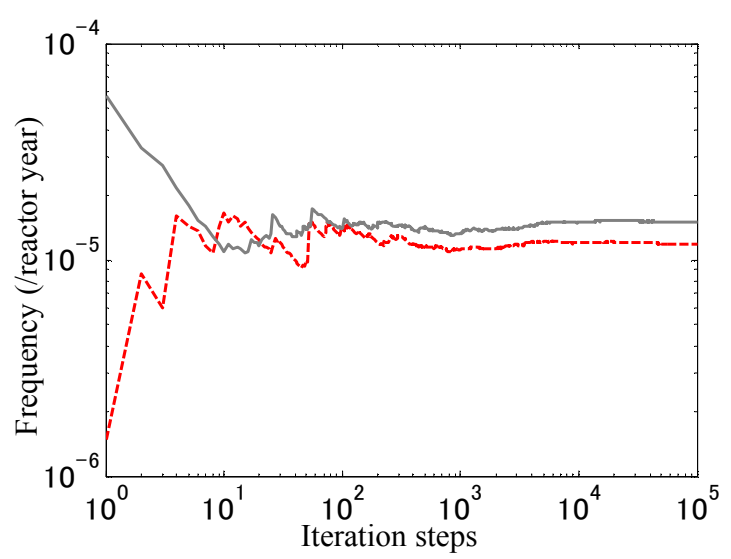

(a) CDF convergence trajectories

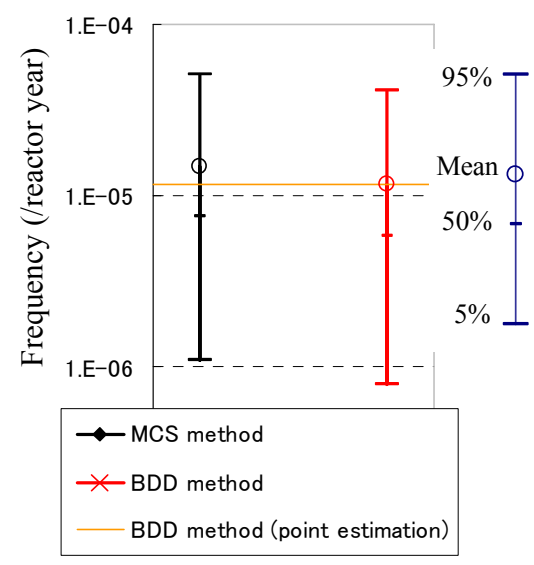

(b) CDF distributions

Fig. 9 The results of uncertainty analysis with the second hazard curve

The total CDFs calculated by both MCS and BDD methods with the second set hazard of curves mostly converged by $10^{4}$ (Fig. 9 (a)) as it was in the previous experiment with the first set of hazard curves. The amounts of time used for MCS and BDD methods were 12,633.4 and 9,809.2 seconds respectively for $10^{5}$ iterations of the Monte Carlo simulations. Therefore, BDD method turned out to be faster than MCS method again. This can be explained by the fact that the size of BDD for this model is smaller than that of MCS.

The CDF distributions obtained with the second hazard curve were shown in Fig. 9 (b). With the results shown in Fig. 9 (b) and Fig. 7 (a) put together, it can be seen that the mean values of CDF computed by BDD method agreed with the values of the point estimations. This should always be true because the value of Eq. (3) and the mean value of Eq. (4) are mathematically equivalent ${ }^{18}$ and because the expected value operator holds multiplicativity for uncorrelated variables and has linear property. These mathematical concepts and the results of this paper suggest that an uncertainty analysis with a mean value much higher 
than the result of point estimation (such as the result shown in table 7 of Ref. (24)) may not have yet reached a certain degree of convergence. Indeed, if the simulations are stopped around $10^{2}$ iterations in the experiments in this paper, the means of CDFs will be highly overestimated (Fig. 7 (b) and Fig. 9 (a)).

Table 3 The ratio of CDFs calculated by MCS method to those by BDD method*

\begin{tabular}{|c|c|c|c|c|c|c|c|c|c|c|c|c|c|}
\hline \multirow{2}{*}{$\begin{array}{c}\text { Seque } \\
\text { nce }\end{array}$} & \multicolumn{6}{|c|}{ The ratio of occurrence frequency } & \multirow{2}{*}{$\begin{array}{c}\text { Seque } \\
\text { nce }\end{array}$} & \multicolumn{6}{|c|}{ The ratio of occurrence frequency } \\
\hline & $\mathrm{PE}$ & Mean & $50 \%$ & $5 \%$ & $95 \%$ & $\mathrm{EF}$ & & $\mathrm{PE}$ & Mean & $50 \%$ & $5 \%$ & $95 \%$ & $\mathrm{EF}$ \\
\hline RPV & 1.00 & 1.00 & 1.00 & 1.00 & 1.00 & 1.00 & S2UW & 0.38 & 0.60 & 0.41 & 0.22 & 0.56 & 1.60 \\
\hline AW & 1.29 & 1.16 & 1.22 & 0.96 & 1.28 & 1.15 & S2UV & 0.77 & 0.81 & 0.48 & 0.34 & 0.75 & 1.49 \\
\hline AUW & 0.53 & 0.80 & 0.28 & 0.03 & 0.77 & 5.44 & S2UX & 1.00 & 1.03 & 0.97 & 0.71 & 1.05 & 1.22 \\
\hline AUV & 1.30 & 1.28 & 0.93 & 0.22 & 1.37 & 2.52 & S2C & 0.62 & 0.80 & 0.42 & 0.46 & 0.67 & 1.21 \\
\hline$A C$ & 0.47 & 0.69 & 0.16 & 0.03 & 0.44 & 3.68 & S2B & 1.37 & 1.16 & 1.24 & 1.20 & 1.17 & 0.99 \\
\hline$A B$ & 1.39 & 1.17 & 1.21 & 1.20 & 1.20 & 1.00 & S2BU1 & 1.55 & 1.26 & 1.35 & 1.24 & 1.29 & 1.02 \\
\hline $\mathrm{ABU}$ & 1.27 & 1.15 & 1.09 & 1.02 & 1.17 & 1.07 & S2BU & 1.61 & 1.34 & 1.44 & 1.51 & 1.33 & 0.94 \\
\hline$A B C$ & 4.45 & 2.77 & 5.49 & 13.10 & 2.82 & 0.46 & S2BC & 5.39 & 2.92 & 9.63 & 15.72 & 3.19 & 0.45 \\
\hline S1W & 1.31 & 1.18 & 1.36 & 1.29 & 1.24 & 0.98 & TW & 1.23 & 1.13 & 1.22 & 1.12 & 1.14 & 1.01 \\
\hline S1UW & 0.57 & 0.84 & 0.58 & 0.23 & 0.85 & 1.92 & TU1W & 1.42 & 1.26 & 1.37 & 1.26 & 1.32 & 1.02 \\
\hline S1UV & 1.31 & 1.30 & 1.32 & 0.80 & 1.38 & 1.32 & TUW & 0.55 & 0.71 & 0.63 & 0.60 & 9 & 1.07 \\
\hline S1UX & 0.71 & 0.86 & 0.67 & 0.42 & 0.85 & 1.41 & TUV & 0.96 & 0.93 & 0.63 & 0.57 & 0.90 & 1.26 \\
\hline S1C & 0.52 & 0.72 & 0.30 & 0.25 & 0.56 & 1.49 & TUX & 1.12 & 1.09 & 1.10 & 1.00 & 1.10 & 1.05 \\
\hline S1B & 1.39 & 1.17 & 1.23 & 1.18 & 1.20 & 1.01 & TC & 1.16 & 1.22 & 1.05 & 1.12 & 1.29 & 1.07 \\
\hline S1BU & 1.37 & 1.20 & 1.20 & 1.17 & 1.21 & 1.02 & TB & 1.29 & 1.12 & 1.19 & 1.10 & 1.13 & 1.01 \\
\hline S1BC & 5.13 & 2.93 & 8.46 & 22.15 & 3.08 & 0.37 & TBU1 & 1.40 & 1.20 & 1.28 & 1.12 & 1.23 & 1.05 \\
\hline $\mathrm{S} 2 \mathrm{~W}$ & 1.15 & 1.1 & 1.1 & 1.0 & 1.13 & 1.04 & TBU & 1.46 & 1.29 & 1.40 & 1.44 & 1.28 & 0.94 \\
\hline S2U1W & 1.44 & 1.28 & 1.46 & 1.38 & 1.36 & 0.99 & TBC & 4.79 & 2.70 & 8.28 & 5.29 & 3.12 & 0.77 \\
\hline & & & & & & & TOTA & 1.52 & 1.26 & 1.30 & 1.38 & 1.24 & 0.95 \\
\hline
\end{tabular}

*In the table, 'PE' means point estimation and 'EF' represents error factor.

Although it turned out that the difference between total CDFs of MCS method and BDD method was not very large (Fig. 7 (a) and Fig. 9 (b)), the disagreement between the methods might not have been negligible in accident sequence level (Table 3). For instance, the error factors (calculated as the square root of the $95 \%$ value divided by the $5 \%$ value) regarding accident sequence AUW, AUV, AC and others shown in Table 3 with gray cells had large variations between MCS and BDD methods. The common property of these sequences is that they are the sequences occurring when Emergency Power Supply (EPS) has succeeded (Fig. 4). To understand this phenomenon, it is required to see what's happening in the sequences for the 'opposite' scenario where EPS fails, which are the accident sequences $\mathrm{ABC}, \mathrm{S} 1 \mathrm{BC}$, and $\mathrm{S} 2 \mathrm{BC}$. The ratios of the error factors for them turned out to be very small because the $5 \%$ values were severely overestimated, but not so much for the $95 \%$ values (Table 3 ) due to the probabilistic limit for overestimation of 1.0 . As a result of the higher 'overestimation' for the 5\% values of accident sequences where EPS fails, the $5 \%$ values for the accident sequences where EPS succeeds were largely 'underestimated', which explains the large deviation of the error factors shown in the figure with gray cells. As one can see from this, the effect of UBA method on uncertainty ranges is attributed not only to its approximation error, but the probabilistic limit of the error. This makes it difficult to predict the effect of approximation error in MCS method.

The effect of the probabilistic limitation on the overestimation of MCS method can also be seen in the comparison between the mean values of the uncertainty analysis and the values of the point estimation (Table 3). However, in this case, the limitation tended to mitigate the approximation error of MCS method. That is, the ratios of mean values obtained by MCS method to those by BDD method were closer to 1.0 than the ratios of the point estimation with the exception of only two sequences (TUV and TC). 


\section{Conclusions}

This paper has examined the accuracy and the computational time of MCS, MC, and BDD methods in uncertainty analysis as well as in point estimation. As to the accuracy, it was confirmed that the results calculated by MC method agrees well with that by BDD method, which is the exact solution, in both point estimation and uncertainty analysis. On the other hand, it was observed that the approximation error of MCS method in point estimation can cause CDFs per accident sequence to be inconsistent in the similar degree with those presented in Ref. (8); while some accident sequences were overestimated, others were underestimated, and hence the rank of dominant accident sequences can be misleading. However, the result of this paper has shown that the inconsistency in using the MCS method abated in the uncertainty analysis since the right tails of overestimated CCDP distributions are limited by the probabilistic upper bound of 1.0. The more essential reason as to why MCS method did not agree was that the MCS method needs to use approximation methods as expressed in Eqs. (3) and (3)'. Therefore, although it is always preferable to use BDD or MC method in terms of accuracy, MCS method may not be as bad of an option in the uncertainty analysis as it is in the point estimate calculation.

As for the computational time, uncertainty analyses in this study required at least $10^{4}$ Monte Carlo iterations to archive a certain degree of convergence for total CDF no matter which method was used. The comparison of the amounts of time used by the three methods has shown that the fastest method was BDD method, the second was MCS method, and the third was MC method. More concretely, while BDD and MCS methods required around 9 hours and 5 hours respectively for $10^{5}$ iterations, MC method spent around 376 hours to perform $10^{4}$ iterations.

Based on the accuracy and the computational time discussed above, BDD method can be considered as the most effective method. However, BDD method is not always applicable to a large Fault-tree and Event-tree as discussed in this paper by referring previous works. Therefore, if BDD method turns out to be inapplicable, or if time is not a serious concern in a PSA study, MC method is a great alternative to be used for both point estimation and uncertainty analysis. In fact, the case where computational time is an extremely huge factor for a PSA study would be almost only when online monitoring of the risk (Risk Monitor) is involved in the study. Therefore, for seismic PSA without online risk monitoring, the applicability of MC method would be very high. Though, because MC method was confirmed to require a lot of time for uncertainty analysis in particular, it may be a good idea to use MCS method to obtain a rough estimation in uncertainty analysis if the error properties of the approximation are carefully considered, such as the effect of probabilistic upper bound on error factors, which was revealed in this paper. Note that acceptable accuracy and computational time vary for different PSA studies and thereby this paper cannot identify one perfect method. Instead, this study provides information for researchers and analysts to select a suitable method in accordance with their acceptable levels for accuracy and computational time.

The points made in this paper were supported by using only one PSA model. However, it is clear that the results of this study depend only on the complex structures of Boolean functions and on the existence of high probabilities for basic events, both of which are common in seismic PRA models for NPPs. Thus, the qualitative implications of the results in this paper are likely to be applicable to almost all seismic PRA models for NPPs and even to other PSA models having such properties. Some major points that are newly reveled in this study are listed in the following as a brief summary.

- MCS method may underestimate accident sequences as well as overestimate them.

- MC (DQFM) method tends to underestimate risks until the enough number of iteration is conduced (see Section 3.2 for more information as to the concrete number).

- MC method agrees well with exact solutions in uncertainty analysis as well as in point 
estimation while MCS method does not.

- For MCS method, in comparison with the approximation error in point estimation, the error in uncertainty analysis tends to be diminished due to the effect of the probabilistic upper bound (1.0).

- It may require $10^{4}$ iterations to reach the point of convergence for uncertainty analysis while Ref. (24) conducted only $10^{3}$ iterations without justification (Ref. (25) showed that $10^{3}$ iterations were enough for uncertainty analysis of a "fragility curve").

- The fastest method was BDD method (around 5 hours), the second was MCS method (around 9 hours), and the third was MC method (around 376 hours).

- Based on the findings above, a way to select a suitable method was discussed (see the third paragraph of this section.)

Given the conclusion above, further developments and studies to speed up MC method and to expand the applicability of BDD method would be beneficial for seismic PSA. Also, it would be worthwhile to examine the efficiencies of each method in the case where correlations among component failures are considered since this study does not cover that area.

Now that the importance of risks due to external events is well recognized, PSA for external events, including earthquakes, will be conducted more intensely in the world. Particularly in Japan, due to the Fukushima Daiichi nuclear accident, the risks of external events other than earthquake will likely start being assessed in the future. However, to gain insights from the assessments in an accurate and efficient manner, an appropriate analytical method needs to be chosen in accordance with the nature of each method and the scope of each PSA study. The results of this study may support the decision-making process to select a proper analytical method not only for seismic PSA but also for other kinds of external PSA because of the computational similarities among them.

\section{Acknowledgement}

The authors would like to thank Dr. Loh of ABS Consulting, Inc. for his careful review of this paper and his support.

\section{References}

(1) U.S. Nuclear Regulatory Commission (USNRC)., "Reactor safety study: An assessment of accident risks in U. S. commercial nuclear power plants", NUREG-75/014 (WASH-1400), (1975).

(2) Sues R. H., Armco, P. J., Campbell, R. D., "Significance of earthquake risk in nuclear power plant probabilistic risk assessments", Nuclear Engineering and Design, Vol.123, No. 1 (1990), pp. 27-44.

(3) The International Atomic Energy Agency (IAEA) ed., "Advanced Nuclear Power Plant Design Options to Cope with External Events", IAEA, IAEA-TECDOC-1487 (2006), pp.57-59.

(4) The Organisation for Economic Co-operation and Development (OECD) ed., "Probabilistic Safety Analysis (PSA) of other External Events than Earthquake", OECD/NEA, NEA/CSNI/R(2009)4.

(5) U.S. Nuclear Regulatory Commission (USNRC), "Individual Plant Examination of External Events (IPEEE) for Severe Accident Vulnerabilities", USNRC, Generic Letter No. 88-20, Supplement 4, (1991).

(6) Chen, J. T., Chokshi, N. C., Kenneally, R. M., Kelly, G. B., Beckner, W. D., McCracken, C., Murphy, A. J., Reiter, L., Jeng, D., "Procedural and Submittal Guidance of Individual Plant Examination of External Events (IPEEE) for Severe Accident Vulnerabilities", NUREG-1407, (1991). 
(7) The American Society of Mechanical Engineers (ASME)., "Addenda to ASME/ANS RA-S-2008, Standard for Level 1/Large Early Release Frequency Probabilistic Risk Assessment for Nuclear Power Plant Applications", ASME/ANS RA-S-2009.

(8) Uchiyama, T., Kawaguchi, K., Wakabayashi, T., "Effect of Analytical Methodology for Assessment on Seismically Induced Core Damage", Journal of Power and Energy Systems, Vol. 5, No. 3 (2011), pp.279-294.

(9) Uchiyama, T., Kawaguchi, K., Wakabayashi, T., "Simultaneous Consideration for Seismically Induced Events on Core Damage Frequency", Journal of Power and Energy Systems, Vol. 5, No. 3 (2011), pp. 360-375.

(10) Epstein, S., Rauzy, A., "Can we trust PRA?", Reliability Engineering and System Safety, Vol. 88, No. 3 (2005), pp. 195-205.

(11) Nusbaumer, O. P. M., "Analytical Solutions of Linked Fault Tree Probabilistic Risk Assessments using Binary Decision Diagrams with Emphasis on Nuclear Safety Applications", ETH Zurich, No. 17286 (2007).

(12) Watanabe, Y., Oikawa, T., Muramatsu, K., "Development of the DQFM method to consider the effect of correlation of component failures in seismic PSA of nuclear power plant", Reliability Engineering and System Safety, Vol. 79, No. 3 (2003), pp. 265-79.

(13) Bedford, T., Cooke MR, "Probabilistic Risk Analysis: Foundations and Methods", (2001), 99-139 \& 326-372, Cambridge University Press.

(14) Chokshi, N., "SPRA Standard and SPRA Uses in the US", presented on 1st Kashiwazaki Symposium on Seismic Safety of Nuclear Installations (2010).

(15) Kennedy, R. P., Cammpbell, R. D., Cornell, C. A., Kaplan, S. and Perla, H., "Probabilistic Seismic Safety Study of an Existing Nuclear Power Plant", Nuclear Engineering and Design, Vol. 59, No. 2 (1980), pp.315-338.

(16) Hwang, H. H. M., "Seismic probabilistic risk assessment and seismic margins studies for nuclear power plants", Probabilistic Engineering Mechanics, Vol. 3, No. 4 (1988), pp. $170-178$.

(17) Hang, Y-N., Whittaker, A. S., Luco, N., "A probabilistic seismic risk assessment procedure for nuclear power plants: (I) Methodology", Nuclear Engineering and Design, Vol. 241, No. 9 (2011), pp. 3996-4003.

(18) Kaplan, S., Bier, V. M., Bley, D. C., "A note on families of fragility curves - is the composite curve equivalent to the mean curve?", Reliability Engineering and System Safety, Vol. 43, No. 3 (1994), pp. 257-261.

(19) H. Kumamoto, K. Tanaka, K. Inoue \& E. J. Henley, "Dagger Sampling Monte Carlo for System Unavailability Evaluation”, IEEE Transactions of Reliability, Vol. R-29, No. 2 (1980), pp. 122-125.

(20) Bryant, R., "Graph Based Algorithms for Boolean Function Manipulation", IEEE Transactions on Computers, Vol. 35, No. 8 (1986), pp. 677-691.

(21) Ibàñez-Llano, C., Rauzy, A., Meléndez, E., Nieto, F., "Hybrid approach for the assessment of PSA models by means of binary decision diagrams", Reliability Engineering and System Safety, Vol. 95, No. 10 (2010), pp. 1076-1092.

(22) Risk Analysis Laboratory, "Summary Report of Seismic PSA of BWR Model Plant", JAERI-Research 99-035, JAERI, Japan, 1999.

(23) EPRI, U.S. DOE, and U.S. NRC, "Technical Report: Central and Eastern United States Seismic Source Characterization for Nuclear Facilities", EPRI, U.S. DOE, and U.S. NRC, 2012.

(24) Hakata, T., "Seismic PSA method for multiple nuclear power plants in a site", Reliability Engineering and System Safety, Vol. 92, No. 7 (2007), pp. 883-894.

(25) Kim, J.H., I.-K. Choi, and J.-H. Park., "Uncertainty analysis of system fragility for seismic safety evaluation of NPP”, Nuclear Engineering and Design, Vol. 241, No. 7 (2011), pp. 2570-2579. 